\title{
Relativistic wave equations of $n$-body systems of fermions and antifermions of various masses in quantum electrodynamics
}

\author{
Mohsen Emami-Razavi ${ }^{1}$, Nantel Bergeron ${ }^{2}$ and Jurij W. Darewych ${ }^{3}$ \\ ${ }^{1}$ Centre for Research in Earth and Space Science, \\ 2 Department of Mathematics and Statistics, \\ 3 Department of Physics and Astronomy, \\ York University, Toronto, Ontario, M3J 1P3, Canada
}

17 Sept 2012

\begin{abstract}
The variational method in a reformulated Hamiltonian formalism of Quantum Electrodynamics is used to derive relativistic wave equations for systems consisting of $n$ fermions and antifermions of various masses. The derived interaction kernels of these equations include onephoton exchange interactions. The equations have the expected Schrödinger non-relativistic limit. Application to some exotic few lepton systems is discussed briefly.
\end{abstract}

\section{Introduction:}

It is straightforward to write down the non-relativistic (Schrödinger) equation for a system of $n$ particles (fermions or bosons), particularly since the interparticle interactions can be adequately described by potentials. Such is not the case for relativistic systems, and a many-body quantized field theory must be used in which the quanta of the interactions are treated on par with the particles. In a recent paper, M. Emami-Razavi derived relativistic $n$-body wave equations of combined fermions and antifermions with equal masses, including the interactions, starting from the underly- 
ing Quantum Electrodynamics (QED) Lagrangian. [1]. A generalization to systems of $n$ fermions and antifermions of different masses is presented in this paper.

Many body fermion systems with electromagnetic interactions are the substance of atomic physics. Fundamental fermion and antifermion systems with electromagnetic interactions are of particular interest because they are "pure" QED systems, with point-like constituents and no nuclear force or size effects. Examples of such systems include positronium (Ps: $\left.e^{+} e^{-}\right)$and muonium $\left(\mathrm{Mu}: \mu^{+} e^{-}\right)$and their ions $\left(\mathrm{Ps}^{-}: e^{+} e^{-} e^{-}, \mathrm{Mu}^{-}: \mu^{+} e^{-} e^{-}\right)$, four body systems such as $\mathrm{Ps}_{2}$ $\left(e^{+} e^{-} e^{+} e^{-}\right), \mathrm{Mu}_{2}\left(\mu^{+} e^{-} \mu^{+} e^{-}\right)$. More generally, for "exotic atoms" such as $e^{+} \mathrm{PsH}$, and $\mathrm{Ps}_{2} \mathrm{O}$, $\mathrm{Li}^{+} \mathrm{Ps}_{2}$ or $\mathrm{Na}^{+} \mathrm{Ps}_{2}$, etc., nuclear size effects are not negligible. These have received attention in the past (e.g. ref. [2]).

The problem of describing relativistic bound states in quantum field theory (QFT) was solved many years ago by Bethe and Salpeter (BS) [3]-4], at least in principle. However, the BS method is not free of complications, such as the existence of relative-time coordinates, difficulty of implementation for systems of more than two bodies, and in practice, the perturbative treatment of interactions. There are many papers available in the literature that use the BS method, at least for two and three body systems. For example, Adkins and co-workers [5]-6] have used Bethe-Salpeter formalism for the calculation of recoil corrections to the energy levels of hydrogenic ions, and a discussion of issues that will have to be treated for the many-electron case, where highly accurate experiments have been carried out, is given. Using a different approach than the BS formalism, Barut [7] summarized his previous work (including work with his co-authors) and generalized his two-body QED equation to many-body particles interacting via the exchange of massless vector bosons. In his formulation [7], the relativistic many-body problem has a structure that is similar to that of the Schrödinger many-body problem.

An alternative to the BS and other approaches is the variational method within the reformulated Hamiltonian formalism of QFT, introduced by Darewych [8]-9]. Among the appealing features of this approach is that it is straightforwardly generalizable to systems of more than two particles, and it can be cast in the form of a relativistic generalization of the Schrödinger description of $n$-body systems. As a variational method it is applicable, at least in principle, to strongly coupled systems 
for which perturbation theory may be unreliable. It has disadvantages as well, particularly in that it may not be manifestly covariant, and like all variational methods, the construction of realistic yet tractable trial states may be a difficult task. A variational approximation is, of course, only as good as the trial states that are being employed. In this paper we use the formalism of [8]-9] to derive relativistic wave equations for systems of $n$ fermions and antifermions of different mass (where $n$ can be any integer number). To our knowledge, there are no papers in the literature that present relativistic wave equations for a system of $n$ fermion with different masses in QED.

Regarding relativistic or QED corrections to the non-relativistic eigenenergies of few body exotic systems we can mention the following. Accurate calculations of the positronium hyperfine structure, i.e. the $O\left(\alpha^{6}\right)$ contributions to ground-state hyperfine splitting in positronium, have been studied by Adkins et al. [10]-[13. Theoretical studies of the $\mathrm{Ps}^{-}\left(e^{-} e^{+} e^{-}\right)$are now well advanced, including perturbative determinations of relativistic and QED corrections (cf. Drake and Grigorescu [14] and references therein.); the leading relativistic and QED corrections to the nonrelativistic ground state energy of the three-body system $\mathrm{Ps}^{-}$have been calculated numerically using a Hylleraas correlated basis set. The corrections to this energy in ref. [14] include the lowest order Breit interaction, the vacuum polarization potential, one and two photon exchange contributions, the annihilation interaction, and spin-spin contact terms. In a recent work, Bubin et al. [15] reported that they have obtained a very accurate variational wave function for non-relativistic binding energy of the positronium molecule $\left(\mathrm{Ps}_{2}, e^{+} e^{-} e^{+} e^{-}\right)$, which they used to calculate the relativistic corrections.

Experiments on such exotic atoms, though difficult, have been and are being undertaken not only for positronium, muonium and the three-body $\mathrm{Ps}^{-}$system, but also for the four-body "positronium molecule" ( $\left.\mathrm{P}_{\mathrm{S}_{2}}: e^{+} e^{-} e^{+} e^{-}\right)$. In 2007, the positronium molecule was observed by Cassidy and Mills [16]. $\mathrm{Ps}_{2}$ creation [16] heralds a new chapter in the study of matter and antimatter and the binding of positrons to ordinary atoms [2]. Another example is the system consisting of three distinct fermions $\left(e^{-}, \tau^{-}, \mu^{+}\right)$[17]. To our knowledge this system has not been observed yet. Other systems of interest are four or more fermions interacting electromagnetically, for example, the system $\left(m^{Z^{+}}, \mathrm{Ps}^{-}\right)$, where $m$ is the mass of a specified particle [18]. The properties of some exotic five-particle systems have been studied in [19]. 
In general, the approach to the calculation of the properties of systems like those cited in the preceding paragraphs has been to calculate accurate solutions of the non-relativistic (Schrödinger) equation then to use these to calculate relativistic and QED corrections to the non-relativistic eigenenergies by means of perturbation theory. We present a method in which the starting point is from relativistic equations that include all "tree-level" interactions. As will be explained below, this approach automatically includes all effects to $O\left(\alpha^{4}\right)$, much like the Dirac equation for the one-electron system in a Coulomb potential, which yields eigenenergies that are correct to $O\left(\alpha^{4}\right)$.

The presentation of this paper is as following. The relativistic n-body QED wave equations for different "flavors" are presented in section II. Two, three, and four-body examples are given in section III. Concluding remarks are presented in section IV.

\section{QED $n$-body wave equations:}

As in previous work [1, we use the variational method in the reformulated version of QED (Darewych [8], Terekidi and Darewych [20]), in which Green's functions are used to express the mediating field in terms of the particle fields.

We start with the usual Lagrangian (density) for $n$ fermions and antifermions, corresponding to fields $\psi_{j}(x)$ and masses $m_{j}(j=1,2,3, \ldots)$, interacting electromagnetically $(\hbar=c=1)$ :

$$
\mathcal{L}=\sum_{j=1 \ldots n} \bar{\psi}_{j}(x)\left(i \gamma^{\mu} \partial_{\mu}-m_{j}-q_{j} \gamma_{\mu} A^{\mu}(x)\right) \psi_{j}(x)-\frac{1}{4} F^{\mu \nu}(x) F_{\mu \nu}(x),
$$

where $x=(t, \mathbf{r}), A^{\mu}(x)$ is the photon field, $F_{\mu \nu}(x)=\partial_{\mu} A_{\nu}(x)-\partial_{\nu} A_{\mu}(x)$, and the coupling constants $q_{j}$ can have arbitrary values. The equations of motion that follow from (11) are

$$
\left(i \gamma^{\mu} \partial_{\mu}-m_{j}\right) \psi_{j}(x)=q_{j} \gamma^{\mu} A_{\mu}(x) \psi_{j}(x)
$$

and

$$
\partial_{\mu} F^{\mu \nu}(x)=j^{\nu}(x)
$$

where

$$
j^{\nu}(x)=\sum_{j=1 \ldots n} q_{j} \bar{\psi}_{j}(x) \gamma^{\nu} \psi_{j}(x) .
$$


As is well known, the Maxwell equation (3) has the formal solution

$$
A_{\mu}(x)=A_{\mu}^{0}(x)+\int d^{4} x^{\prime} D_{\mu \nu}\left(x-x^{\prime}\right) j^{\nu}\left(x^{\prime}\right)
$$

where $A_{\mu}^{0}(x)$ is a solution of the homogeneous ("free field") equation (3) (i.e. with $j^{\nu}(x)=0$ ) and $D_{\mu \nu}\left(x-x^{\prime}\right)$ is the a Green function (or "photon propagator"), such that

$$
\partial_{\alpha} \partial^{\alpha} D_{\mu \nu}\left(x-x^{\prime}\right)-\partial_{\mu} \partial^{\alpha} D_{\alpha \nu}\left(x-x^{\prime}\right)=g_{\mu \nu} \delta^{4}\left(x-x^{\prime}\right)
$$

Substitution of the formal solution (5) into (2) yields the result

$$
\left(i \gamma^{\mu} \partial_{\mu}-m_{j}\right) \psi_{j}(x)=q_{j} \gamma^{\mu}\left(A_{\mu}^{0}(x)+\int d^{4} x^{\prime} D_{\mu \nu}\left(x-x^{\prime}\right) j^{\nu}\left(x^{\prime}\right)\right) \psi_{j}(x) .
$$

Equations (17) are derivable from the stationary action principle $\delta \int d^{4} x \mathcal{L}_{R}(x)=0$, where

$$
\mathcal{L}_{R}=\sum_{j=1 \ldots n} \bar{\psi}_{j}(x)\left(i \gamma^{\mu} \partial_{\mu}-m_{j}-q_{j} \gamma^{\mu} A_{\mu}^{0}(x)\right) \psi_{j}(x)-\frac{1}{2} \int d^{4} x^{\prime} j^{\mu}\left(x^{\prime}\right) D_{\mu \nu}\left(x-x^{\prime}\right) j^{\nu}(x),
$$

provided that the Green function $D_{\mu \nu}\left(x-x^{\prime}\right)$ is symmetric.

The Hamiltonian density corresponding to the Lagrangian (8) is obtained using the usual canonical prescription

$$
\mathcal{H}=\sum_{j} \pi_{\psi_{j}} \dot{\psi}_{j}-\mathcal{L}_{R}, \quad \text { where } \quad \pi_{\psi_{j}}=\frac{\partial \mathcal{L}_{R}}{\partial \dot{\psi}_{j}}=i \psi_{j}^{\dagger} .
$$

This yields the result

$$
\mathcal{H}(x)=\mathcal{H}_{0}(x)+\mathcal{H}_{I_{1}}(x)+\mathcal{H}_{I_{2}}(x),
$$

where

$$
\begin{gathered}
\mathcal{H}_{0}(x)=\sum_{j=1 \ldots n} \psi_{j}^{\dagger}(x)\left(-i \vec{\alpha} \cdot \nabla+m_{j} \beta\right) \psi_{j}(x), \\
\mathcal{H}_{I_{1}}(x)=\sum_{j=1 \ldots n} q_{j} \bar{\psi}_{j}(x) \gamma^{\mu} A_{\mu}^{0}(x) \psi_{j}(x), \\
\mathcal{H}_{I_{2}}(x)=\frac{1}{2} \int d^{4} x^{\prime} j^{\mu}\left(x^{\prime}\right) D_{\mu \nu}\left(x-x^{\prime}\right) j^{\nu}(x)
\end{gathered}
$$

with $j^{\nu}(x)=\sum_{j=1 \ldots n} q_{j} \bar{\psi}_{j}(x) \gamma^{\nu} \psi_{j}(x)$ and $D_{\mu \nu}\left(x-x^{\prime}\right)=\int \frac{1}{(2 \pi)^{4}} d^{4} k D_{\mu \nu}(k) \exp \left(-i k\left(x-x^{\prime}\right)\right)$. 
We shall consider only stationary bound-states of $n$-fermion systems (or their scattering) in this work. Thus we shall not consider processes that involve the emission or absorption of free (physical) photons in the present work. Moreover, the variational trial states that we use (see eqs. (20) and (21)) do not sample the term corresponding to eq. (12), i.e the matrix elements of $H_{I_{1}}$ with respect to the trial states (201) and (21)) vanish. Consequently we shall leave out the free photon field $A_{\mu}^{0}(x)$ in what follows. Thus, the Hamiltonian density that we shall work with, namely $\mathcal{H}(x)=\mathcal{H}_{0}(x)+\mathcal{H}_{I_{2}}(x)$, contains the Fermion fields and the photon propagators $D_{\mu \nu}$ only. Nevertheless, one should note that virtual photons are included in our work, by virtue of the photon propagator in the interaction term, eq. (13).

In practice, a choice of gauge is needed to specify the Green function. The covariant Lorentz gauge $\partial_{\mu} A^{\mu}=0$ is a frequent choice. For bound-state problems the Coulomb gauge is a convenient choice. In momentum representation it is

$$
D_{00}(k)=\frac{1}{|\mathbf{k}|^{2}}, \quad D_{0 j}(k)=0, \quad D_{i j}(k)=\frac{1}{k^{\mu} k_{\mu}}\left(\delta_{i j}-\frac{k_{i} k_{j}}{\mathbf{k}^{2}}\right) .
$$

We construct a quantum field theory, based on the reformulated Hamiltonian, by promoting the field variables to operators which satisfy the usual (anti)commutation rules. Furthermore, we use the usual Fourier decomposition

$$
\psi_{j}(x)=\sum_{s} \int \frac{d^{3} p}{(2 \pi)^{3 / 2}}\left(\frac{m_{j}}{\omega_{j p}}\right)^{1 / 2}\left[b_{j}(\mathbf{p}, s) u_{j}(\mathbf{p}, s) e^{-i p \cdot x}+d_{j}^{\dagger}(\mathbf{p}, s) v_{j}(\mathbf{p}, s) e^{i p \cdot x}\right],
$$

where $p=p^{\mu}=\left(\omega_{j p}, \mathbf{p}\right)$, and $\omega_{j p}=\sqrt{m_{j}^{2}+\mathbf{p}^{2}}$; that is, the field operators $\psi_{j}$ (and their conjugates $\psi_{j}^{\dagger}$ ) are replaced by linear superpositions of "ladder" operators $b_{j}, d_{j}^{\dagger}$ (and their conjugates $\left.b_{j}^{\dagger}, d_{j}\right)$. The mass- $m_{j}$ free-particle Dirac spinors $u_{j}$ and $v_{j}$, where $\left(\gamma^{\mu} p_{\mu}-m_{j}\right) u_{j}(\mathbf{p}, s)=0$, $\left(\gamma^{\mu} p_{\mu}+m_{j}\right) v_{j}(\mathbf{p}, s)=0$, are normalized such that

$$
u_{j}^{\dagger}(\mathbf{p}, s) u_{j}(\mathbf{p}, \sigma)=v_{j}^{\dagger}(\mathbf{p}, s) v_{j}(\mathbf{p}, \sigma)=\frac{\omega_{j p}}{m_{j}} \delta_{s \sigma}, \quad \text { with } u_{j}^{\dagger}(\mathbf{p}, s) v_{j}(\mathbf{p}, \sigma)=v_{j}^{\dagger}(\mathbf{p}, s) u_{j}(\mathbf{p}, \sigma)=0 .
$$

The creation and annihilation operators $b^{\dagger}, b$ of the (free) fermions of mass $m_{j}$, and $d^{\dagger}, d$ for the corresponding antiparticles, satisfy the usual anticommutation rules. The non-vanishing ones are

$$
\left\{b_{j}(\mathbf{p}, s), b_{j}^{\dagger}(\mathbf{q}, \sigma)\right\}=\left\{d_{j}(\mathbf{p}, s), d_{j}^{\dagger}(\mathbf{q}, \sigma)\right\}=\delta_{s \sigma} \delta^{3}(\mathbf{p}-\mathbf{q}) .
$$


The vacuum state $|0\rangle$ is defined by $b_{j}(\mathbf{p}, s)|0\rangle=d_{j}(\mathbf{p}, s)|0\rangle=0$. Note that this is a "filled negativeenergy sea" vacuum, as is clear from the fact that the eigenvalues of $\hat{H}_{0}$ are positive and there are no "negative-energy" solutions.

With the replacement (15), and its conjugate for $\psi_{j}^{\dagger}$, the Hamiltonian operator, $\hat{H}=\int d^{3} x \hat{\mathcal{H}}(x)$ is expressed in terms of the creation and annihilation operators $b^{\dagger}, d^{\dagger}, b, d$ in the usual way. Note that we normal-order the entire Hamiltonian (thereby denoting it $: \hat{H}:)$, since this circumvents the need for mass renormalization which would otherwise arise. Not that there is a difficulty with handling mass renormalization in the present formalism, as shown in various earlier papers (see, for example, 20]). It is simply that we are not interested in mass renormalization here, since it has no effect on bound state energies. Furthermore, the approximate trial states which we use in this work, are incapable of sampling loop effects. Thus, the normal ordering of the entire Hamiltonian does not sweep under the carpet loop renormalization effects, since none arise at the present level of approximation.

The Hamiltonian operator, specifically its interaction part $\hat{H}_{I_{2}}$, is time dependent (the free-field part, $\hat{H}_{0}$, is time-independent). For the description of stationary states it is convenient to work with a time-independent Hamiltonian. This can be achieved by a unitary transformation of states and operators by means of the unitary operator $e^{i H_{0} t}$. The result is the same as setting $t=0$ in all matrix elements below, which we henceforth do. We might mention that if we do not set $t=0$, we would have extra factors of the form the of $e^{i\left(\omega_{j p_{j}^{\prime}}+\omega_{k p_{k}^{\prime}}-\omega_{j p_{j}}-\omega_{k p_{k}}\right) t}$ in the matrix elements of $H_{I_{2}}$; but, as already stated, this "phase factor" time dependence can be eliminated (rotated away to unity) by a unitary transformation or, equivalently, setting $t=0$.

In the Hamiltonian formalism of QFT we seek solutions of the eigenvalue equation.

$$
\hat{P}^{\beta}|\Psi\rangle=Q^{\beta}|\Psi\rangle
$$

where $\hat{P}^{\beta}=(\hat{H}, \hat{\mathbf{P}})$ is the energy-momentum operator of the QFT, and $Q^{\beta}=(E, \mathbf{Q})$ is the energymomentum eigenvalue. The case $\mathbf{Q}=0$ defines the rest-frame of the system. The $\beta=0$ component of (18) is generally not solvable, hence approximation methods, such as the variational method, must 
be used. The latter amounts to finding approximate solutions by using the variational principle

$$
\delta\left\langle\Psi_{t r}|: \hat{H}-E:| \Psi_{t r}\right\rangle_{t=0}=0
$$

where $\left|\Psi_{t r}\right\rangle$ is a suitably chosen trial state.

We consider two explicit types of $n$-fermion systems, one with an equal number of particles and antiparticles and one with the number of particles greater by one that the number of antiparticles. For a system of $\frac{n}{2}$ particles and $\frac{n}{2}$ antiparticles (here $n$ is even) the simplest trial state is

$\left|\Psi_{n}\right\rangle=\sum_{s_{1} \ldots s_{n}} \int d^{3} p_{1} \ldots d^{3} p_{n} F_{s_{1} s_{2} \ldots s_{n}}\left(\mathbf{p}_{1}, \ldots, \mathbf{p}_{n}\right) b_{1}^{\dagger}\left(\mathbf{p}_{1}, s_{1}\right) d_{2}^{\dagger}\left(\mathbf{p}_{2}, s_{2}\right) \ldots . b_{n-1}^{\dagger}\left(\mathbf{p}_{n-1}, s_{n-1}\right) d_{n}^{\dagger}\left(\mathbf{p}_{n}, s_{n}\right)|0\rangle$,

where the functions $F_{s_{1} \ldots s_{n}}\left(\mathbf{p}_{1}, \ldots, \mathbf{p}_{n}\right)$ are well-behaved, adjustable function (normalizable for bound states $) ; s_{j}, \mathbf{p}_{j}(j=1, . . n)$ are the spin indices and momentum coordinates of the fermions that make up the $n$-body system. Similarly, for a system of $\frac{n+1}{2}$ particles and $\frac{n-1}{2}$ antiparticles (here $n$ is odd) we have

$$
\left|\Psi_{n}\right\rangle=\sum_{s_{1} \ldots s_{n}} \int d^{3} p_{1} \ldots d^{3} p_{n} F_{s_{1} \ldots s_{n}}\left(\mathbf{p}_{1}, \ldots, \mathbf{p}_{n}\right) b_{1}^{\dagger}\left(\mathbf{p}_{1}, s_{1}\right) d_{2}^{\dagger}\left(\mathbf{p}_{2}, s_{2}\right) \ldots d_{n-1}^{\dagger}\left(\mathbf{p}_{n-1}, s_{n-1}\right) b_{n}^{\dagger}\left(\mathbf{p}_{n}, s_{n}\right)|0\rangle .
$$

The variational coefficient functions $F_{s_{1} \ldots s_{n}}\left(\mathbf{p}_{1}, \ldots, \mathbf{p}_{n}\right)$ will be determined in accordance with the variational principle (19). Not all of them will be independent since they must be chosen to be eigenstates of the total momentum and angular momentum (magnitude and projection) of the field theory.

The trial states (20) and (21) are variational approximations to the unknown exact eigenstates of the Hamiltonian. They are constructed as superpositions of the eigenstates of the free Hamiltonian $H_{0}$, equation (11), the so-called "Fock-states". This is analogous to expanding an eigenfunction $\phi(\mathbf{r})$ of a one-particle system in Schrödinger quantum mechanics in terms of the free-particle states $e^{i \mathbf{p} \cdot \mathbf{r}}$, i.e. $\phi(\mathbf{r})=\int d^{3} p f(\mathbf{p}) e^{i \mathbf{p} \cdot \mathbf{r}}$ (which would be the exact eigensolution with an appropriate choice of $f(\mathbf{p}))$. Of course, in QFT the state (20) or (21) cannot be an exact eigenstate of the full Hamiltonian, no matter what choice of $F_{s_{1} \ldots s_{n}}\left(\mathbf{p}_{1}, \ldots, \mathbf{p}_{n}\right)$ is made. Rather, they are approximate (or "trial") states. 
To implement the variational principle (19) we must calculate the matrix elements of the Hamiltonian operator using the trial states (20), (21). The matrix element corresponding to the restplus-kinetic energy of such a $n$-fermion system is

$$
\left\langle\Psi_{n}\left|: \hat{H}_{\psi}-E:\right| \Psi_{n}\right\rangle=\sum_{s_{1} \ldots s_{n}} \int d^{3} p_{1} \ldots d^{3} p_{n} F_{s_{1} \ldots s_{n}}^{*}\left(\mathbf{p}_{1}, \ldots, \mathbf{p}_{n}\right) F_{s_{1} \ldots s_{n}}\left(\mathbf{p}_{1}, \ldots, \mathbf{p}_{n}\right)\left[\omega_{1 p_{1}}+\cdots+\omega_{n p_{n}}-E\right] .
$$

The matrix element corresponding to the interactions is a sum of terms corresponding to attractive one photon exchange (plus repulsive virtual annihilation interactions for each particle-antiparticle pair, if there were any) and repulsive one photon exchange between pairs of fermions of the same sign of charge. Symbolically,

$$
\left\langle\Psi_{n}\left|: \hat{H}_{I}:\right| \Psi_{n}\right\rangle=\left[\mathcal{M}^{\text {Attractive }}\right]+\left[\mathcal{M}^{\text {Repulsive }}\right]
$$

If $n$ is even (i.e. an equal number of particles and antiparticles), there are $n^{2} / 4$ particle-antiparticle combinations and $\left(n^{2}-2 n\right) / 4$ two-identical-charged-fermion combinations. For example, for $n=4$, we have 4 attractive one-photon exchange terms, 2 repulsive one-photon exchange terms. If $n$ is odd (i.e. one more particle than antiparticle) there are $\left(n^{2}-1\right) / 4$ attractive and $(n-1)^{2} / 4$ repulsive terms. For example, for $n=5$, we have 6 attractive, 4 repulsive terms.

For the $n$-body system described by the trial state (20) or (21), the matrix element corresponding to the interactions is $\left\langle\Psi_{n}\left|: \hat{H}_{I}:\right| \Psi_{n}\right\rangle=\left\langle\Psi_{n}\left|: \hat{H}_{I_{2}}:\right| \Psi_{n}\right\rangle$, since $\left\langle\Psi_{n}\left|: \hat{H}_{I_{1}}:\right| \Psi_{n}\right\rangle=0$. That is, as stated previously, the variational trial states (20) or (21) do not sample the term : $\hat{H}_{I_{1}}$ : (cf. eq. (12)) of the interaction Hamiltonian. To repeat, this means that with such simple trial states only stationary (stable bound or scattering) states can be described, but not processes that involve the 
emission or absorption of physical photons. Thus,

$$
\begin{aligned}
& \left\langle\Psi_{n}\left|\quad: \quad \hat{H}_{I} \quad:\right| \Psi_{n}\right\rangle=\left\langle\Psi_{n}\left|: \quad \hat{H}_{I_{2}} \quad:\right| \Psi_{n}\right\rangle \\
& =\sum_{\substack{s_{1} \ldots s_{n} \\
s_{1}^{\prime} \ldots s_{n}^{\prime}}} \int d^{3} p_{1} \ldots d^{3} p_{n} d^{3} p_{1}^{\prime} \ldots d^{3} p_{n}^{\prime} F_{s_{1}^{\prime} s_{2}^{\prime} \ldots s_{n}^{\prime}}^{*}\left(\mathbf{p}_{1}^{\prime}, \ldots \mathbf{p}_{n}^{\prime}\right) F_{s_{1} s_{2} \ldots s_{n}}\left(\mathbf{p}_{1}, \ldots, \mathbf{p}_{n}\right) \\
& \left\{\sum_{j=1}^{n-1} \sum_{k=j+1}^{n}\left[\prod_{i=1 \ldots n}^{(j, k)} \delta_{s_{i}^{\prime} s_{i}}\right]\left[\prod_{i=1 \ldots n}^{(j, k)} \delta^{3}\left(\mathbf{p}_{i}^{\prime}-\mathbf{p}_{i}\right)\right] \frac{m_{j} m_{k} q_{j} q_{k}}{2(2 \pi)^{3}} \frac{\delta^{3}\left(\mathbf{p}_{j}^{\prime}+\mathbf{p}_{k}^{\prime}-\mathbf{p}_{j}-\mathbf{p}_{k}\right)}{\sqrt{\omega_{j p_{j}^{\prime}} \omega_{k p_{k}^{\prime}} \omega_{j p_{j} \omega_{k p_{k}}}} \times}\right. \\
& \left(-\mathcal{M}_{s_{j} s_{k} s_{j}^{\prime} s_{k}^{\prime}}^{\text {Attractive }}\left(\mathbf{p}_{j}, \mathbf{p}_{k}, \mathbf{p}_{j}^{\prime}, \mathbf{p}_{k}^{\prime}\right)\right)+\sum_{j=1}^{n-2} \sum_{k=j+2}^{n}\left[\prod_{i=1 \ldots n}^{(j, k)} \delta_{s_{i}^{\prime} s_{i}}\right]\left[\prod_{i=1 \ldots n}{ }^{(j, k)} \delta^{3}\left(\mathbf{p}_{i}^{\prime}-\mathbf{p}_{i}\right)\right] \times \\
& \left.\frac{m_{j} m_{k} q_{j} q_{k}}{2(2 \pi)^{3}} \frac{\delta^{3}\left(\mathbf{p}_{j}^{\prime}+\mathbf{p}_{k}^{\prime}-\mathbf{p}_{j}-\mathbf{p}_{k}\right)}{\sqrt{\omega_{j p_{j}^{\prime}} \omega_{k p_{k}^{\prime}} \omega_{j p_{j}} \omega_{k p_{k}}}} \mathcal{M}_{s_{j} s_{k} s_{j}^{\prime} s_{k}^{\prime}}^{\text {Repulsive }}\left(\mathbf{p}_{j}, \mathbf{p}_{k}, \mathbf{p}_{j}^{\prime}, \mathbf{p}_{k}^{\prime}\right)\right\}
\end{aligned}
$$

where $\sum_{k=a}^{\prime} u_{k}$ means $u_{a}+u_{a+2}+u_{a+4}+\cdots$. Our convention is that variables with odd indices correspond to particles (e.g. $e^{-}$), and those with even indices correspond to antiparticles (e.g. $\left.e^{+}\right)$.

The superscript notation $(j, k)$ in $\prod_{i=1 \ldots n}^{(j, k)} \delta^{3}\left(\mathbf{p}_{i}^{\prime}-\mathbf{p}_{i}\right)$ means that the terms with indices $j$ and $k$ are left out:

$$
\prod_{i=1 . . n}^{(j, k)} \delta^{3}\left(\mathbf{p}_{i}^{\prime}-\mathbf{p}_{i}\right)=\prod_{i=1}^{j-1} \delta^{3}\left(\mathbf{p}_{i}^{\prime}-\mathbf{p}_{i}\right) \prod_{i=j+1}^{k-1} \delta^{3}\left(\mathbf{p}_{i}^{\prime}-\mathbf{p}_{i}\right) \prod_{i=k+1}^{n} \delta^{3}\left(\mathbf{p}_{i}^{\prime}-\mathbf{p}_{i}\right)=\frac{\prod_{i=1}^{n} \delta^{3}\left(\mathbf{p}_{i}^{\prime}-\mathbf{p}_{i}\right)}{\delta^{3}\left(\mathbf{p}_{j}^{\prime}-\mathbf{p}_{j}\right) \delta^{3}\left(\mathbf{p}_{k}^{\prime}-\mathbf{p}_{k}\right)},
$$

and similarly for $\prod_{i=1 \ldots n}{ }^{(j, k)} \delta_{s_{i}^{\prime} s_{i}}$ we have

$$
\prod_{i=1 \ldots n}^{(j, k)} \delta_{s_{i}^{\prime} s_{i}}=\prod_{i=1}^{j-1} \delta_{s_{i}^{\prime} s_{i}} \prod_{i=j+1}^{k-1} \delta_{s_{i}^{\prime} s_{i}} \prod_{i=k+1}^{n} \delta_{s_{i}^{\prime} s_{i}}=\frac{\prod_{i=1}^{n} \delta_{s_{i}^{\prime} s_{i}}}{\delta_{s_{j}^{\prime} s_{j}} \delta_{s_{k}^{\prime} s_{k}}}
$$

For the case $n=2, \prod_{i=1 \ldots n}{ }^{(j, k)} \delta^{3}\left(\mathbf{p}_{i}^{\prime}-\mathbf{p}_{i}\right)=1$ and $\prod_{i=1 \ldots n}{ }^{(j, k)} \delta_{s_{i}^{\prime} s_{i}}=1$. But, for example, for $n=4$ (i.e. a four-body system), we have six terms. We write equations (25) and (26) explicitly for say, $j=2, k=3$.

$$
\begin{aligned}
\prod_{i=1 \ldots 4}{ }^{(2,3)} \delta^{3}\left(\mathbf{p}_{i}^{\prime}-\mathbf{p}_{i}\right) & =\frac{\prod_{i=1}^{4} \delta^{3}\left(\mathbf{p}_{i}^{\prime}-\mathbf{p}_{i}\right)}{\delta^{3}\left(\mathbf{p}_{2}^{\prime}-\mathbf{p}_{2}\right) \delta^{3}\left(\mathbf{p}_{3}^{\prime}-\mathbf{p}_{3}\right)}=\frac{\delta^{3}\left(\mathbf{p}_{1}^{\prime}-\mathbf{p}_{1}\right) \delta^{3}\left(\mathbf{p}_{2}^{\prime}-\mathbf{p}_{2}\right) \delta^{3}\left(\mathbf{p}_{3}^{\prime}-\mathbf{p}_{3}\right) \delta^{3}\left(\mathbf{p}_{4}^{\prime}-\mathbf{p}_{4}\right)}{\delta^{3}\left(\mathbf{p}_{2}^{\prime}-\mathbf{p}_{2}\right) \delta^{3}\left(\mathbf{p}_{3}^{\prime}-\mathbf{p}_{3}\right)} \\
& =\delta^{3}\left(\mathbf{p}_{1}^{\prime}-\mathbf{p}_{1}\right) \delta^{3}\left(\mathbf{p}_{4}^{\prime}-\mathbf{p}_{4}\right)
\end{aligned}
$$




$$
\prod_{i=1 \ldots 4}^{(2,3)} \delta_{s_{i}^{\prime} s_{i}}=\frac{\prod_{i=1}^{4} \delta_{s_{i}^{\prime} s_{i}}}{\delta_{s_{2}^{\prime} s_{2}} \delta_{s_{3}^{\prime} s_{3}}}=\frac{\delta_{s_{1}^{\prime} s_{1}} \delta_{s_{2}^{\prime} s_{2}} \delta_{s_{3}^{\prime} s_{3}} \delta_{s_{4}^{\prime} s_{4}}}{\left.\delta_{s_{2}^{\prime} s_{2}} \delta_{s_{3}^{\prime} s_{3}}\right)}=\delta_{s_{1}^{\prime} s_{1}} \delta_{s_{4}^{\prime} s_{4}}
$$

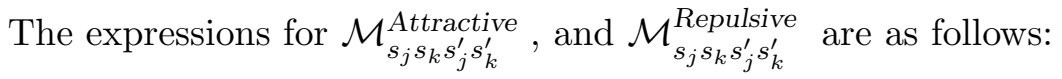

$$
\begin{aligned}
\mathcal{M}_{s_{j} s_{k} s_{j}^{\prime} s_{k}^{\prime}}^{\text {Attractive }}\left(\mathbf{p}_{j}, \mathbf{p}_{k}, \mathbf{p}_{j}^{\prime}, \mathbf{p}_{k}^{\prime}\right)= & \bar{u}_{j}\left(\mathbf{p}_{j}^{\prime}, s_{j}^{\prime}\right) \gamma^{\mu} u_{j}\left(\mathbf{p}_{j}, s_{j}\right)\left[D_{\mu \nu}\left(\omega_{j p_{j}^{\prime}}-\omega_{j p_{j}}, \mathbf{p}_{j}^{\prime}-\mathbf{p}_{j}\right)\right. \\
& \left.+D_{\mu \nu}\left(\omega_{k p_{k}^{\prime}}-\omega_{k p_{k}}, \mathbf{p}_{k}^{\prime}-\mathbf{p}_{k}\right)\right] \bar{v}_{k}\left(\mathbf{p}_{k}, s_{k}\right) \gamma^{\nu} v_{k}\left(\mathbf{p}_{k}^{\prime}, s_{k}^{\prime}\right),
\end{aligned}
$$

if $j$ is odd $\left(\right.$ e.g. $\left.e^{-}\right)$and $k$ is even $\left(\right.$ e.g. $\left.\mu^{+}\right)$, and a similar expression, with $u$ replaced by $v$, and $v$ replaced by $u$ in equation (29) if $j$ is even (i.e. $\mu^{+}$) and $k$ is odd (i.e. $e^{-}$). The terms corresponding to one photon exchange interactions among particles with same sign of charge are

$$
\begin{aligned}
\mathcal{M}_{s_{j} s_{k} s_{j}^{\prime} s_{k}^{\prime}}^{\text {Repulsive }}\left(\mathbf{p}_{j}, \mathbf{p}_{k}, \mathbf{p}_{j}^{\prime}, \mathbf{p}_{k}^{\prime}\right)= & \bar{u}_{j}\left(\mathbf{p}_{j}^{\prime}, s_{j}^{\prime}\right) \gamma^{\mu} u_{j}\left(\mathbf{p}_{j}, s_{j}\right)\left[D_{\mu \nu}\left(\omega_{j p_{j}^{\prime}}-\omega_{j p_{j}}, \mathbf{p}_{j}^{\prime}-\mathbf{p}_{j}\right)\right. \\
& \left.+D_{\mu \nu}\left(\omega_{k p_{k}^{\prime}}-\omega_{k p_{k}}, \mathbf{p}_{k}^{\prime}-\mathbf{p}_{k}\right)\right] \bar{u}_{k}\left(\mathbf{p}_{k}^{\prime}, s_{k}^{\prime}\right) \gamma^{\nu} u_{k}\left(\mathbf{p}_{k}, s_{k}\right),
\end{aligned}
$$

if $j$ and $k$ are both odd (i.e. $e^{-} \mu^{-}$) and a similar expression, with $u$ replaced by $v$ in equation (30) if $j$ and $k$ are both even (i.e. $e^{+} \mu^{+}$). Note that equations (29) and (30) correspond to onephoton exchange Feynman diagrams between any two particles in the system, where (29) applies to particles of opposite sign and (30) to particles of the same sign.

The relativistic $n$-body wave equations for the coefficient functions $F_{s_{1} s_{2} \ldots s_{n}}\left(\mathbf{p}_{1}, \ldots, \mathbf{p}_{n}\right)$ of the trial state (20) or (21) that follows from $\delta\left\langle\Psi_{n}|: \hat{H}-E:| \Psi_{n}\right\rangle_{t=0}=0$, is

$$
\begin{aligned}
& F_{s_{1} s_{2} \ldots s_{n}}\left(\mathbf{p}_{1}, \ldots, \mathbf{p}_{n}\right)\left[\omega_{1 p_{1}}+\cdots+\omega_{n p_{n}}-E\right]=\sum_{s_{1}^{\prime} \ldots s_{n}^{\prime}} \int d^{3} p_{1}^{\prime} \ldots d^{3} p_{n}^{\prime} F_{s_{1}^{\prime} s_{2}^{\prime} \ldots s_{n}^{\prime}}\left(\mathbf{p}_{1}^{\prime}, \ldots, \mathbf{p}_{n}^{\prime}\right) \\
& \left\{\sum_{j=1}^{n-1} \sum_{k=j+1}^{n}\left[\prod_{i=1 \ldots n}^{(j, k)} \delta_{s_{i}^{\prime} s_{i}}\right]\left[\prod_{i=1 \ldots n}^{(j, k)} \delta^{3}\left(\mathbf{p}_{i}^{\prime}-\mathbf{p}_{i}\right)\right] \frac{m_{j} m_{k} q_{j} q_{k}}{2(2 \pi)^{3}} \frac{\delta^{3}\left(\mathbf{p}_{j}^{\prime}+\mathbf{p}_{k}^{\prime}-\mathbf{p}_{j}-\mathbf{p}_{k}\right)}{\sqrt{\omega_{j p_{j}^{\prime}} \omega_{k p_{k}^{\prime}} \omega_{j p_{j}} \omega_{k p_{k}}}} \times\right. \\
& \mathcal{M}_{s_{j} s_{k} s_{j}^{\prime} s_{k}^{\prime}}^{\text {Attractive }}\left(\mathbf{p}_{j}, \mathbf{p}_{k}, \mathbf{p}_{j}^{\prime}, \mathbf{p}_{k}^{\prime}\right)-\sum_{j=1}^{n-2} \sum_{k=j+2}^{n}\left[\prod_{i=1 \ldots n}{ }^{(j, k)} \delta_{s_{i}^{\prime} s_{i}}\right]\left[\prod_{i=1 \ldots n}{ }^{(j, k)} \delta^{3}\left(\mathbf{p}_{i}^{\prime}-\mathbf{p}_{i}\right)\right] \frac{m_{j} m_{k} q_{j} q_{k}}{2(2 \pi)^{3}} \times \\
& \left.\frac{\delta^{3}\left(\mathbf{p}_{j}^{\prime}+\mathbf{p}_{k}^{\prime}-\mathbf{p}_{j}-\mathbf{p}_{k}\right)}{\sqrt{\omega_{j p_{j}^{\prime}} \omega_{k p_{k}^{\prime}} \omega_{j p_{j}} \omega_{k p_{k}}}} \mathcal{M}_{s_{j} s_{k} s_{j}^{\prime} s_{k}^{\prime}}^{\text {Repulsive }}\left(\mathbf{p}_{j}, \mathbf{p}_{k}, \mathbf{p}_{j}^{\prime}, \mathbf{p}_{k}^{\prime}\right)\right\}
\end{aligned}
$$

where $\sum_{k=a}^{\prime} u_{k}$ means $u_{a}+u_{a+2}+u_{a+4}+\cdots$, as before. 
Equation (31) is our main result. It is a relativistic momentum-space equation for stationary (stable bound or scattering) states of a $n$-fermion system, consisting of the same number of differentmass fermions and antifermions if $n$ is even or with the number of particles one larger than the number of antiparticles (or vice versa) if $n$ is odd, but with no particle-antiparticle pairs. It is Salpeter-like (Schrödinger-like) in structure, with positive-energy solutions only, as can be seen by setting the right-hand-side of eq. (31) to zero, whereupon $E=\sum_{j} \omega_{j p_{j}}>0$ (as it must be given our use of the "filled negative-energy" vacuum $|0\rangle$, defined below eq. (17)). In this respect eq. (31) is different from many-fermion Dirac-like equations or Bethe-Salpeter equations, which do have negative-energy solutions (these, however, are generally disregarded in studies of two or more body bound state systems).

One should note that the equations (31) derived in the present article are relativistic equations in which the kinematics of the $n$-fermion system with arbitrary masses are treated exactly and the centre of mass motion is taken into account without any approximation, that is the trial state (20) or (21) is an eigenstate of the total momentum operator $\hat{\mathbf{P}}$ of the field theory with eigenvalue $\mathbf{Q}$ (see eq. (18)). [Q can be taken to be zero in the rest frame of the system.] Thus the mass polarization is built into the relativistic equations since no assumptions about any of the particle masses being infinitely heavy are made. There is no need for perturbative expansions in mass ratios.

The interaction kernels (relativistic momentum space potentials) in eq. (31) contain only treelevel Feynman diagrams (cf. (29), (30)), that is, one-quantum exchange, including retardation effects. This means that physical effects to $O\left(\alpha^{4}\right)$ only are contained in eq. (31). (This has been shown explicitly for the two and three fermion systems in earlier studies, 21] for positronium, [20] for muonium and [17] for $e^{+} e^{-} e^{-}$and $\mu^{+} e^{-} e^{-}$, as discussed in more detail in section 3 below.) To include effects beyond $O\left(\alpha^{4}\right)$ requires the use of more elaborate approximations than just the simplest trial states (20) and (21), as has been illustrated on the relatively simple case of positronium (cf. ref. [22]). Alternatively, one could "cheat" by simply adding matrix elements corresponding to higher-order (loop) diagrams to the kernels (29) and (30).

To help understand the content of the approximations inherent in equation (31) it is useful to consider its non-relativistic limit, i.e. when $\mathbf{p}^{2} / m^{2}<<1$. In this limit $D_{\mu \nu}$ in the expressions for 
$\mathcal{M}_{s_{j} s_{k} s_{j}^{\prime} s_{k}^{\prime}}^{\text {Attractive }}$ and $\mathcal{M}_{s_{j} s_{k} s_{j}^{\prime} s_{k}^{\prime}}^{\text {Repulsive }}$ reduce to $D_{00}=1 /|\mathbf{q}-\mathbf{p}|^{2}$ (and zero otherwise) for the attractive and repulsive terms. Thus, in the non relativistic limit,

$$
\tilde{\mathcal{M}}_{s_{j} s_{k} s_{j}^{\prime} s_{k}^{\prime}}^{\text {Attractive }}=2 \bar{u}\left(0, s_{j}^{\prime}\right) \gamma^{0} u\left(0, s_{j}\right) \frac{1}{\left|\mathbf{p}_{j}^{\prime}-\mathbf{p}_{j}\right|^{2}} \bar{v}\left(0, s_{k}\right) \gamma^{0} v\left(0, s_{k}^{\prime}\right)=\frac{2 \delta_{s_{j} s_{j}^{\prime}} \delta_{s_{k} s_{k}^{\prime}}}{\left|\mathbf{p}_{j}^{\prime}-\mathbf{p}_{j}\right|^{2}}
$$

and

$$
\tilde{\mathcal{M}}_{s_{j} s_{k} s_{j}^{\prime} s_{k}^{\prime}}^{\text {Repulsive }}=2 \bar{u}\left(0, s_{j}^{\prime}\right) \gamma^{0} u\left(0, s_{j}\right) \frac{1}{\left|\mathbf{p}_{j}^{\prime}-\mathbf{p}_{j}\right|^{2}} \bar{u}\left(0, s_{k}^{\prime}\right) \gamma^{0} u\left(0, s_{k}\right)=\frac{2 \delta_{s_{j} s_{j}^{\prime}} \delta_{s_{k} s_{k}^{\prime}}}{\left|\mathbf{p}_{j}^{\prime}-\mathbf{p}_{j}\right|^{2}} .
$$

For arbitrary $n$, the coordinate-space equation, obtained by Fourier transformation

$$
F_{s_{1} s_{2} \ldots s_{n}}\left(\mathbf{p}_{1}, \ldots, \mathbf{p}_{n}\right)=\frac{1}{(2 \pi)^{3 n / 2}} \int d^{3} x_{1} \ldots d^{3} x_{n} \Psi_{s_{1} s_{2} \ldots s_{n}}\left(\mathbf{x}_{1}, \ldots, \mathbf{x}_{n}\right) e^{-i\left(\mathbf{p}_{1} \cdot \mathbf{x}_{1}+\cdots+\mathbf{p}_{n} \cdot \mathbf{x}_{n}\right)}
$$

of the non-relativistic limit of eq. (31), is as expected, the $n$-body Schrödinger equation,

$$
\left[-\sum_{i=1}^{n} \frac{1}{2 m_{i}} \nabla_{i}^{2}-\epsilon-\sum_{j=1}^{n-1} \sum_{k=j+1}^{n}{ }^{\prime} \frac{\alpha_{j k}}{\left|\mathbf{x}_{j}-\mathbf{x}_{k}\right|}+\sum_{j=1}^{n-2} \sum_{k=j+2}^{n}{ }^{\prime} \frac{\alpha_{j k}}{\left|\mathbf{x}_{j}-\mathbf{x}_{k}\right|}\right] \Psi_{s_{1} \ldots s_{n}}\left(\mathbf{x}_{1}, \ldots, \mathbf{x}_{n}\right)=0
$$

where $\epsilon=E-\left[m_{1}+m_{2}+\cdots+m_{n}\right], \alpha_{j k}=\frac{q_{j} q_{k}}{4 \pi}$ are the coupling constants, and $\Sigma_{k=a}{ }^{\prime} u_{k}$ means $u_{a}+u_{a+2}+u_{a+4}+\cdots$.

In the non-relativistic limit, we see that the same equations are obtained for all $\Psi_{s_{1} \ldots s_{n}}$ (or, equivalently, for all $F_{s_{1} \ldots s_{n}}$ ), hence the spin and space parts of the non-relativistic wave functions separate, and we can write $\Psi_{s_{1} s_{2} \ldots s_{n}}\left(\mathbf{x}_{1}, \ldots, \mathbf{x}_{n}\right)=\Lambda_{s_{1} s_{2} \ldots s_{n}} \Psi\left(\mathbf{x}_{1}, \ldots, \mathbf{x}_{n}\right)$. The $\Lambda_{s_{1} s_{2} \ldots s_{n}}$ are spin coefficients that must be chosen so that $\sum_{s_{1} \ldots s_{n}} F_{s_{1} \ldots s_{n}}$ is the appropriate $n$-body angular-momentum eigenstate.

The variationally obtained $n$-body relativistic equations (31) (or non-relativistic equations (35) for $n>2$ ) are not analytically solvable, hence approximate, usually variational, solutions must be obtained. Thus, for any $n$ and any state, one can obtain an approximate variational solution for the energy $E_{n}$ and the wave-function of the $n$-body system by replacing $F_{s_{1} s_{2} \ldots s_{n}}\left(\mathbf{p}_{1}, \ldots, \mathbf{p}_{n}\right)$ with analytic functions containing adjustable features (parameters) to compute the energy expectation value (see Eqs. (22)-(24)):

$$
E_{n}=\frac{\left\langle\Psi_{n}|: \hat{H}:| \Psi_{n}\right\rangle}{\left\langle\Psi_{n} \mid \Psi_{n}\right\rangle}
$$


Optimal values of the adjustable features (parameters) of the trial wave functions correspond to the minimum values of (36). This minimum principle allows for a systematic improvement of the approximate (variational) solutions.

\section{Two, three and four-body examples:}

The relativistic equations (31) have been previously derived and solved approximately for the $n=2$, two-body cases (like muonium, $\mu^{+} e^{-}$, ref. [20]) and the $n=3$, three-body cases (like $\mu^{+} e^{-} e^{-}$, ref. [17]). It was shown that results, correct to $O\left(\alpha^{4}\right)$, are obtained for the energies of all bound states of these systems. We shall recount some details.

Thus, for the two-body problem $\left(e^{-} \mu^{+}\right)$, for which the trial state (20) is

$$
\left|\Psi_{2}\right\rangle=\sum_{s_{1} s_{2}} \int d^{3} p_{1} d^{3} p_{2} F_{s_{1} s_{2}}\left(\mathbf{p}_{1}, \mathbf{p}_{2}\right) b_{1}^{\dagger}\left(\mathbf{p}_{1}, s_{1}\right) d_{2}^{\dagger}\left(\mathbf{p}_{2}, s_{2}\right)|0\rangle,
$$

the wave equation (31) becomes:

$$
\begin{aligned}
& F_{s_{1} s_{2}}\left(\mathbf{p}_{1}, \mathbf{p}_{2}\right)\left[\omega_{1 p_{1}}+\omega_{2 p_{2}}-E\right]=\frac{q_{1} q_{2} m_{1} m_{2}}{2(2 \pi)^{3}} \sum_{s_{1}^{\prime} s_{2}^{\prime}} \int d^{3} p_{1}^{\prime} d^{3} p_{2}^{\prime} F_{s_{1}^{\prime} s_{2}^{\prime}}\left(\mathbf{p}_{1}^{\prime}, \mathbf{p}_{2}^{\prime}\right) \frac{\delta^{3}\left(\mathbf{p}_{1}^{\prime}+\mathbf{p}_{2}^{\prime}-\mathbf{p}_{1}-\mathbf{p}_{2}\right)}{\sqrt{\omega_{1 p_{1}^{\prime}} \omega_{2 p_{2}^{\prime}} \omega_{1 p_{1}} \omega_{2 p 2}}} \\
& \times\left[\mathcal{M}_{s_{1} s_{2} s_{1}^{\prime} s_{2}^{\prime}}^{\text {Attractive }}\left(\mathbf{p}_{1}, \mathbf{p}_{2}, \mathbf{p}_{1}^{\prime}, \mathbf{p}_{2}^{\prime}\right)\right] \\
& \mathcal{M}_{s_{1} s_{2} s_{1}^{\prime} s_{2}^{\prime}}^{\text {Attractive }}\left(\mathbf{p}_{1}, \mathbf{p}_{2}, \mathbf{p}_{1}^{\prime}, \mathbf{p}_{2}^{\prime}\right)= \bar{u}_{1}\left(\mathbf{p}_{1}^{\prime}, s_{1}^{\prime}\right) \gamma^{\mu} u_{1}\left(\mathbf{p}_{1}, s_{1}\right)\left[D_{\mu \nu}\left(\omega_{1 p_{1}^{\prime}}-\omega_{1 p_{1}}, \mathbf{p}_{1}^{\prime}-\mathbf{p}_{1}\right)\right. \\
&\left.+D_{\mu \nu}\left(\omega_{2 p_{2}^{\prime}}-\omega_{2 p_{2}}, \mathbf{p}_{2}^{\prime}-\mathbf{p}_{2}\right)\right] \bar{v}_{2}\left(\mathbf{p}_{2}, s_{2}\right) \gamma^{\nu} v_{2}\left(\mathbf{p}_{2}^{\prime}, s_{2}^{\prime}\right),
\end{aligned}
$$

(Of course, there is no repulsive term for $\left(e^{-} \mu^{+}\right)$case.) Equations (38) and (39) were derived previously [20]. We mention, in passing, that for a system consisting a particle and antiparticle of equal mass (like positronium) an additional virtual annihilation interaction term, $\mathcal{M}_{s_{j} s_{k} s_{j}^{\prime} s_{k}^{\prime}}^{\text {Annion }}$ arises in Eq. (38) as shown in [21]:

$$
\begin{aligned}
\mathcal{M}_{s_{1} s_{2} s_{1}^{\prime} s_{2}^{\prime}}^{\text {Annian }}\left(\mathbf{p}_{1}, \mathbf{p}_{2}, \mathbf{p}_{1}^{\prime}, \mathbf{p}_{2}^{\prime}\right)= & \bar{u}\left(\mathbf{p}_{1}^{\prime}, s_{1}^{\prime}\right) \gamma^{\mu} v\left(\mathbf{p}_{2}^{\prime}, s_{2}^{\prime}\right)\left[D_{\mu \nu}\left(\omega_{p_{1}^{\prime}}+\omega_{p_{2}^{\prime}}, \mathbf{p}_{1}^{\prime}+\mathbf{p}_{2}^{\prime}\right)\right. \\
& \left.+D_{\mu \nu}\left(-\omega_{p_{1}}-\omega_{p_{2}},-\mathbf{p}_{1}-\mathbf{p}_{2}\right)\right] \bar{v}\left(\mathbf{p}_{2}, s_{2}\right) \gamma^{\nu} u\left(\mathbf{p}_{1}, s_{1}\right) .
\end{aligned}
$$


The variational two-fermion wave equations for muonium like systems were solved approximately by Terekidi and Darewych [20]; their results were shown to be in agreement with other calculations and in good agreement with the observed muonium spectrum to $\mathrm{O}\left(\alpha^{4}\right)$ (as well as that for hydrogen and muonic hydrogen).

The non-relativistic limit of the wave equation (38) for the two-body system $\left(e^{-} \mu^{+}\right)$is

$$
\begin{aligned}
& F_{s_{1} s_{2}}\left(\mathbf{p}_{1}, \mathbf{p}_{2}\right)\left[\frac{\mathbf{p}_{1}^{2}}{2 m_{1}}+\frac{\mathbf{p}_{2}^{2}}{2 m_{2}}-\epsilon_{2}\right] \\
= & \frac{q_{1} q_{2}}{(2 \pi)^{3}} \sum_{s_{1}^{\prime} s_{2}^{\prime}} \int d^{3} p_{1}^{\prime} d^{3} p_{2}^{\prime} F_{s_{1}^{\prime} s_{2}^{\prime}}\left(\mathbf{p}_{1}^{\prime}, \mathbf{p}_{2}^{\prime}\right) \delta^{3}\left(\mathbf{p}_{1}^{\prime}+\mathbf{p}_{2}^{\prime}-\mathbf{p}_{1}-\mathbf{p}_{2}\right)\left[\frac{\delta_{s_{1} s_{1}^{\prime}} \delta_{s_{2} s_{2}^{\prime}}}{\left|\mathbf{p}_{1}^{\prime}-\mathbf{p}_{1}\right|^{2}}\right],
\end{aligned}
$$

where $\epsilon_{2}=E-\left[m_{1}+m_{2}\right]$. The coordinate-space form of eq. (41), is, of course, equation (35) with $n=2$, namely the expected Schrödinger equation,

$$
\left[-\sum_{i=1}^{2} \nabla_{i}^{2} \frac{1}{2 m_{i}}-\frac{\alpha}{\left|\mathbf{x}_{1}-\mathbf{x}_{2}\right|}-\epsilon_{2}\right] \Psi_{s_{1} s_{2}}\left(\mathbf{x}_{1}, \mathbf{x}_{2}\right)=0
$$

where $\alpha=\frac{q_{1} q_{2}}{4 \pi}$ is the usual fine-structure constant and $\left|q_{1}\right|=\left|q_{2}\right|=|e|$, where $e$ is the elementary charge.

For the general case of three constituents with different masses, systems like $\left(m_{1}^{-} m_{2}^{+} m_{3}^{-}\right)$, with the trial state (cf. eq. (21)),

$$
\left|\Psi_{3}\right\rangle=\sum_{s_{1} s_{2} s_{3}} \int d^{3} p_{1} d^{3} p_{2} d^{3} p_{3} F_{s_{1} s_{2} s_{3}}\left(\mathbf{p}_{1}, \mathbf{p}_{2}, \mathbf{p}_{3}\right) b_{1}^{\dagger}\left(\mathbf{p}_{1}, s_{1}\right) d_{2}^{\dagger}\left(\mathbf{p}_{2}, s_{2}\right) b_{3}^{\dagger}\left(\mathbf{p}_{3}, s_{3}\right)|0\rangle,
$$

the three-body wave equation is (31) with $n=3$, namely

$$
\begin{aligned}
& F_{s_{1} s_{2} s_{3}}\left(\mathbf{p}_{1}, \mathbf{p}_{2}, \mathbf{p}_{3}\right)\left[\omega_{1 p_{1}}+\omega_{2 p_{2}}+\omega_{3 p_{3}}-E\right]=\sum_{s_{1}^{\prime} s_{2}^{\prime} s_{3}^{\prime}} \int d^{3} p_{1}^{\prime} d^{3} p_{2}^{\prime} d^{3} p_{3}^{\prime} F_{s_{1}^{\prime} s_{2}^{\prime} s_{3}^{\prime}}\left(\mathbf{p}_{1}^{\prime}, \mathbf{p}_{2}^{\prime}, \mathbf{p}_{3}^{\prime}\right) \times \\
& \left\{\frac{m_{1} m_{2} q_{1} q_{2}}{2(2 \pi)^{3}} \mathcal{M}_{s_{1} s_{2} s_{1}^{\prime} s_{2}^{\prime}}^{\text {Atractive }}\left(\mathbf{p}_{1}, \mathbf{p}_{2}, \mathbf{p}_{1}^{\prime}, \mathbf{p}_{2}^{\prime}\right) \delta_{s_{3}^{\prime} s_{3}} \delta^{3}\left(\mathbf{p}_{3}^{\prime}-\mathbf{p}_{3}\right) \frac{\delta^{3}\left(\mathbf{p}_{1}^{\prime}+\mathbf{p}_{2}^{\prime}-\mathbf{p}_{1}-\mathbf{p}_{2}\right)}{\sqrt{\omega_{1 p_{1}^{\prime} \omega_{2 p_{2}^{\prime}} \omega_{1 p_{1}} \omega_{2 p_{2}}}}}\right. \\
& +\frac{m_{2} m_{3} q_{2} q_{3}}{2(2 \pi)^{3}} \mathcal{M}_{s_{2} s_{3} s_{2}^{\prime} s_{3}^{\prime}}^{\text {Attractive }}\left(\mathbf{p}_{2}, \mathbf{p}_{3}, \mathbf{p}_{2}^{\prime}, \mathbf{p}_{3}^{\prime}\right) \delta_{s_{1}^{\prime} s_{1}} \delta^{3}\left(\mathbf{p}_{1}^{\prime}-\mathbf{p}_{1}\right) \frac{\delta^{3}\left(\mathbf{p}_{2}^{\prime}+\mathbf{p}_{3}^{\prime}-\mathbf{p}_{2}-\mathbf{p}_{3}\right)}{\sqrt{\omega_{2 p_{2}^{\prime}} \omega_{3 p_{3}^{\prime} \omega_{2 p_{2}} \omega_{3 p_{3}}}}}
\end{aligned}
$$

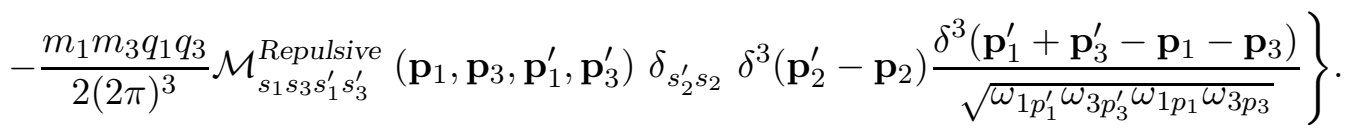




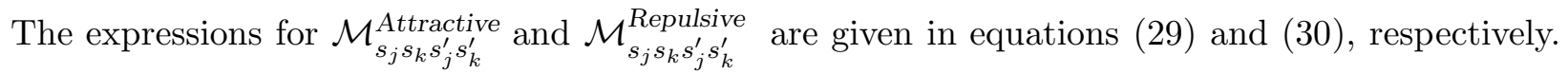
Note that for $\mathrm{H}^{-}$, and the muonium negative ion, $\mathrm{Mu}^{-}\left(e^{-} \mu^{+} e^{-}\right)$, we have the same wave equation (44) but with two of the constituents with equal masses. Note again that $\left|q_{1}\right|=\left|q_{2}\right|=\left|q_{3}\right|=|e|$, where $e$ is the elementary charge. As already mentioned, approximate solutions of the relativistic three fermion equation (44) for some systems are presented in ref. [17]. They are in agreement with other calculations and with experimental results to $O\left(\alpha^{4}\right)$.

In the non-relativistic limit, eq. (44) reduces to the following:

$$
\begin{aligned}
& F_{s_{1} s_{2} s_{3}}\left(\mathbf{p}_{1}, \mathbf{p}_{2}, \mathbf{p}_{3}\right)\left[\frac{\mathbf{p}_{1}^{2}}{2 m_{1}}+\frac{\mathbf{p}_{2}^{2}}{2 m_{2}}+\frac{\mathbf{p}_{3}^{2}}{2 m_{3}}-\epsilon_{3}\right]=\sum_{s_{1}^{\prime} s_{2}^{\prime} s_{3}^{\prime}} \int d^{3} p_{1}^{\prime} d^{3} p_{2}^{\prime} d^{3} p_{3}^{\prime} F_{s_{1}^{\prime} s_{2}^{\prime} s_{3}^{\prime}}\left(\mathbf{p}_{1}^{\prime}, \mathbf{p}_{2}^{\prime}, \mathbf{p}_{3}^{\prime}\right) \\
& \left\{\frac{q_{1} q_{2}}{(2 \pi)^{3}} \frac{\delta_{s_{1} s_{1}^{\prime}} \delta_{s_{2} s_{2}^{\prime}} \delta_{s_{3}^{\prime} s_{3}}}{\left|\mathbf{p}_{1}^{\prime}-\mathbf{p}_{1}\right|^{2}} \delta^{3}\left(\mathbf{p}_{3}^{\prime}-\mathbf{p}_{3}\right) \delta^{3}\left(\mathbf{p}_{1}^{\prime}+\mathbf{p}_{2}^{\prime}-\mathbf{p}_{1}-\mathbf{p}_{2}\right)+\frac{q_{2} q_{3}}{(2 \pi)^{3}} \frac{\delta_{s_{1}^{\prime} s_{1}} \delta_{s_{2} s_{2}^{\prime}} \delta_{s_{3}^{\prime} s_{3}}}{\left|\mathbf{p}_{2}^{\prime}-\mathbf{p}_{2}\right|^{3}\left(\mathbf{p}_{1}^{\prime}-\mathbf{p}_{1}\right) \times}\right. \\
& \left.\delta^{3}\left(\mathbf{p}_{2}^{\prime}+\mathbf{p}_{3}^{\prime}-\mathbf{p}_{2}-\mathbf{p}_{3}\right)-\frac{q_{1} q_{3}}{(2 \pi)^{3}} \frac{\delta_{s_{1}^{\prime} s_{1}} \delta_{s_{2} s_{2}^{\prime}} \delta_{s_{3}^{\prime} s_{3}}}{\left|\mathbf{p}_{1}^{\prime}-\mathbf{p}_{1}\right|^{2}} \delta^{3}\left(\mathbf{p}_{2}^{\prime}-\mathbf{p}_{2}\right) \delta^{3}\left(\mathbf{p}_{1}^{\prime}+\mathbf{p}_{3}^{\prime}-\mathbf{p}_{1}-\mathbf{p}_{3}\right)\right\},
\end{aligned}
$$

where $\epsilon_{3}=E-\left[m_{1}+m_{2}+m_{3}\right]$. In the coordinate space, this becomes the three-body Schrödinger equation (35), with $n=3$, namely

$$
\left\{-\left(\sum_{i=1}^{3} \frac{1}{2 m_{i}} \nabla_{i}^{2}\right)-\epsilon_{3}-\frac{\alpha_{12}}{\left|\mathbf{x}_{1}-\mathbf{x}_{2}\right|}-\frac{\alpha_{23}}{\left|\mathbf{x}_{2}-\mathbf{x}_{3}\right|}+\frac{\alpha_{13}}{\left|\mathbf{x}_{1}-\mathbf{x}_{3}\right|}\right\} \Psi_{s_{1} s_{2} s_{3}}\left(\mathbf{x}_{1}, \mathbf{x}_{2}, \mathbf{x}_{3}\right)=0
$$

where $\alpha_{j k}=\frac{q_{j} q_{k}}{4 \pi}$, as expected.

For the four-body case of various "flavors" $\left(m_{1}^{-} m_{2}^{+} m_{3}^{-} m_{4}^{+}\right)$the relativistic equation is (31) with 
$n=4$, that is

$$
\begin{aligned}
& F_{s_{1} s_{2} s_{3} s_{4}}\left(\mathbf{p}_{1}, \mathbf{p}_{2}, \mathbf{p}_{3}, \mathbf{p}_{4}\right)\left[\omega_{1 p_{1}}+\omega_{2 p_{2}}+\omega_{3 p_{3}}+\omega_{4 p_{4}}-E\right] \\
& =\sum_{s_{1}^{\prime} s_{2}^{\prime} s_{3}^{\prime} s_{4}^{\prime}} \int d^{3} p_{1}^{\prime} d^{3} p_{2}^{\prime} d^{3} p_{3}^{\prime} d^{3} p_{4}^{\prime} F_{s_{1}^{\prime} s_{2}^{\prime} s_{3}^{\prime} s_{4}^{\prime}}\left(\mathbf{p}_{1}^{\prime}, \mathbf{p}_{2}^{\prime}, \mathbf{p}_{3}^{\prime}, \mathbf{p}_{4}^{\prime}\right) \times
\end{aligned}
$$

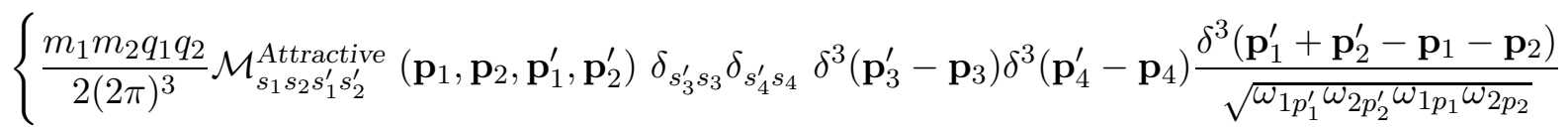

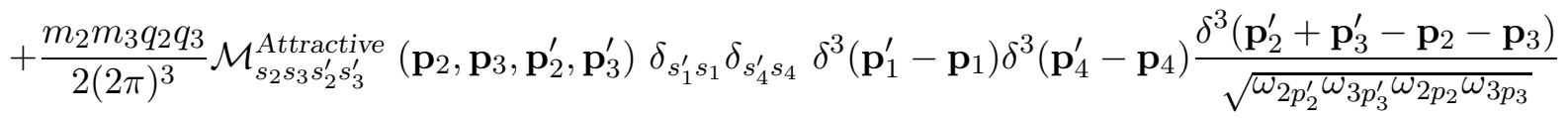

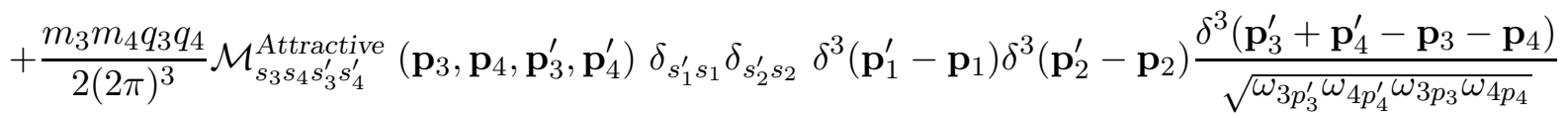

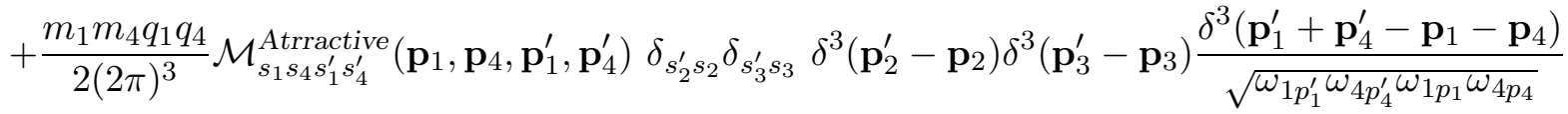

$$
\begin{aligned}
& -\frac{m_{1} m_{3} q_{1} q_{3}}{2(2 \pi)^{3}} \mathcal{M}_{s_{1} s_{3} s_{1}^{\prime} s_{3}^{\prime}}^{\text {Repulise }}\left(\mathbf{p}_{1}, \mathbf{p}_{3}, \mathbf{p}_{1}^{\prime}, \mathbf{p}_{3}^{\prime}\right) \delta_{s_{2}^{\prime} s_{2}} \delta_{s_{4}^{\prime} s_{4}} \delta^{3}\left(\mathbf{p}_{2}^{\prime}-\mathbf{p}_{2}\right) \delta^{3}\left(\mathbf{p}_{4}^{\prime}-\mathbf{p}_{4}\right) \frac{\delta^{3}\left(\mathbf{p}_{1}^{\prime}+\mathbf{p}_{3}^{\prime}-\mathbf{p}_{1}-\mathbf{p}_{3}\right)}{\sqrt{\omega_{1 p_{1}^{\prime}} \omega_{3 p_{3}^{\prime}} \omega_{1 p_{1}} \omega_{3 p_{3}}}}
\end{aligned}
$$

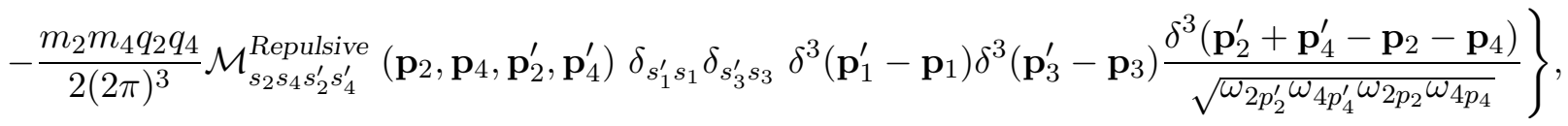

The expressions for $\mathcal{M}_{s_{j} s_{k} s_{j}^{\prime} s_{k}^{\prime}}^{\text {Attractive }}$ and $\mathcal{M}_{s_{j} s_{k} s_{j}^{\prime} s_{k}^{\prime}}^{\text {Repulsive }}$ are given in equations (29) and (30). No (approximate) solutions of the relativistic four-fermion equation (47) have been obtained to date. The non-relativistic limit of (47) is, of course, equation (35) with $n=4$.

One can, analogously, write out the explicit expression for the relativistic $n$-fermion equation (31) for $n=5$ or larger.

\section{Concluding remarks.}

The solution of the non-relativistic $n$-body system is a difficult problem for $n>3$; all the more so for the relativistic counterpart. Theoretical investigation of non-relativistic four-body systems interacting through Coulombic potentials have been discussed in several works and studies have been done regarding the existence of bound-states of such systems, including the domain of stability in the space of inverse masses [23]. Some exotic, non-relativistic five-body systems have been investigated by Mezei et al. [19], who studied the stability of a number of five-body systems using 
stochastic variational method (SVM). However, much remains to be done in the field of relativistic equations (including QED effects) for atomic $n$-body systems with $n \geq 3$, particularly, systems of different masses. Equation (31) can be used to calculate relativistic effects, though the effort required is considerable even in the case of weak binding, when perturbation theory with respect to (approximate) non-relativistic solutions is applicable.

One should note that the systems considered in this paper are basically composed of fundamental fermions, such as electron $(e)$, muon $(\mu)$, or tauon $(\tau)$, and their corresponding antifermions. During the early days of quantum mechanics a suitable set of coordinates and basis states for the three-body problem was proposed by Hylleraas [24], and it was used to calculate the ground state energy of the helium atom. At that time (1929) the proton was considered to be a fundamental ("point") particle (protons are now known to have three-quark plus gluon substructure). Around half a century after the work of Hylleraas 24]-[25], some theoretical and experimental advances have been made and extensive high-precision calculations became feasible (see Drake [26]-[28]). Moreover, we can also mention the following example, which illustrates that some unresolved QED problems are under investigation currently. One of the intriguing questions which remain to be answered in bound-state quantum electrodynamics is related to the discrepancy between the theoretical and experimental values for the Lamb shift in ionized helium, or $\mathrm{He}^{+}$, which is a hydrogen like atomic system with a nuclear charge number $\mathrm{Z}=2$; the current status of the subject is discussed in ref. [29].

The situation is different for exotic atoms or ions that are "pure" QED systems, such as the positronium negative ion $\left(\mathrm{Ps}^{-}\right)$or the muonium negative ion $\left(\mathrm{Mu}^{-}\right)$, bound only by electromagnetic interactions. To our knowledge, the binding energy of $\mathrm{Ps}^{-}$or $\mathrm{Mu}^{-}$has not been measured to date. The only four-body exotic system that is a "pure" QED system and which has been observed is the positronium molecule [16]. However, the experimental value of the binding energy has not as yet been obtained for $\mathrm{Ps}_{2}$. The situation is even worse for systems of $n>3$ fermions of various mass; for example, $\mu^{+} e^{-} \mu^{+} e^{-}$has not even been observed as yet, and to our knowledge there is no relativistic or QED study of this system $\left(\mathrm{Mu}_{2}\right)$. Therefore, we can say that we are still in the early stage of study of these exotic pure QED systems. Clearly, the study of pure QED systems, bound only by electromagnetic interactions, is of fundamental interest as was shown for the positronium 
$\left(e^{-} e^{+}\right)$system in some recent experiments [30]-31].

To sum up, we have derived relativistic equations (31) for systems of $n \geq 2$ fermions of various mass. The relativistic kinematics are included exactly in equations (31), but the interactions contain tree-level interactions only (one photon exchange, and virtual annihilation in the case of pairs), i.e. they are incomplete beyond $\mathrm{O}\left(\alpha^{4}\right)$. To calculate effects beyond this order, the matrix elements $\mathcal{M}$ in (31) can be augmented by higher order (loop) diagram contributions, as is done in the Bethe-Salpeter formalism (beyond the ladder approximation).

Because our method of deriving the equations (including the interactions) is variational, the description of $n$ fermion system can be improved systematically by using more elaborate $n$-body trial states than (20) and (21), as was done for the two-body Ps system [22]. Variational trial states, such as (20) and (21), can be generalized in various ways. Thus, the single Fock trial state $\left|\Psi_{2}\right\rangle$ (37) can be replaced by a superposition of two (or more) Fock states (as was done for scalar models [32-34]), including states that accommodate virtual pairs. We illustrate this on the two-fermion $\left(\mu^{+} \mu^{-}\right)$system. The single Fock trial state $\left|\Psi_{t}\right\rangle=\left|\Psi_{2}\right\rangle$, where

$$
\left|\Psi_{2}\right\rangle=\sum_{s_{1} s_{2}} \int d^{3} p_{1} d^{3} p_{2} F_{s_{1} s_{2}}\left(p_{1}, p_{2}\right) b_{\mu}^{\dagger}\left(p_{1}, s_{1}\right) d_{\mu}^{\dagger}\left(p_{2}, s_{2}\right)|0\rangle
$$

can be generalized to $\left|\Psi_{t}\right\rangle=\left|\Psi_{2}\right\rangle+\left|\Psi_{4}\right\rangle$, where

$$
\left|\Psi_{4}\right\rangle=\sum_{s_{1} \ldots s_{4}} \int d^{3} p_{1} \ldots d^{3} p_{4} G_{s_{1} \ldots s_{4}}\left(p_{1} \ldots p_{4}\right) b_{\mu}^{\dagger}\left(p_{1}, s_{1}\right) d_{\mu}^{\dagger}\left(p_{2}, s_{2}\right) b_{e}^{\dagger}\left(p_{3}, s_{3}\right) d_{e}^{\dagger}\left(p_{4}, s_{4}\right)|0\rangle,
$$

where $b_{e}^{\dagger}$ and $d_{e}^{\dagger}$ are electron and positron creation operators. The variational principle (19) would then lead to coupled, multi-dimensional integral equations for the channel wave-functions $F$ and $G$. The channel function $G$, evidently, accommodates an electron-positron pair, which is virtual when the system energy domain is $E<2 m_{\mu}$. Clearly the coupled multidimensional equations for $F$ and $G$ can only be solved approximately, say variationally, but this is a tedious calculation that will be left for the future.

It should be mentioned that numerical calculations have been done in order to solve approximately relativistic wave equations of $n$-body systems of scalar particles and antiparticle of various masses (for example ref. [35], for $n=3$ ). However, determining approximate solutions of relativistic 
wave equations for $n$-body systems $(n \geq 3)$ of fermions and antifermions of various masses is considerably more difficult and remains a challenging task. As mentioned previously, it is not possible to solve the relativistic $n$-body equations derived in this paper analytically. Therefore, approximate (i.e., numerical, variational or perturbative) solutions must be sought for various cases of interest. The stochastic variational and quantum Monte Carlo methods are popular among methods for the computation of complicated exotic systems [36]-[37]. In Ref. [38], the possible production of systems such as true muonium $\left(\mu^{+} \mu^{-}\right)$, true tauonium $\left(\tau^{+} \tau^{-}\right)$, and "mu-tauonium" $\left(\mu^{ \pm} \tau^{\mp}\right)$ has been discussed. The discovery of $\left(\mu^{+} \mu^{-}\right)$in future will herald a new chapter for the observation

of much more difficult systems such as $\left(\mu^{ \pm} \tau^{\mp}\right)$. In any case, experiments on other exotic atoms or molecules, though difficult, will be undertaken in future even though it may not be the near future.

Lastly, we wish to point out that it would be of interest to apply the variational method and the Hamiltonian formalism used in the present work to QCD systems in order to derive relativistic, momentum space integral equations for systems consisting of $n$ quarks and anti quarks, interacting via gluon exchange, as has been attempted in a previous paper for a quark-antiquark system [39].

\section{References}

[1] M. Emami-Razavi, Phys. Rev. A 77, 042104 (2008).

[2] J. Mitroy, M. W. Bromley, and G. G. Ryzhikh, J. Phys. B 35, R81-R116 (2002).

[3] E. E. Salpeter and H. A. Bethe, Phys. Rev. 841232 (1951).

[4] H. A. Bethe and E. E. Salpeter, Quantum Mechanics of One- and Two-Electron Atoms, 1957 (Berlin: Springer).

[5] G. S. Adkins, S. Morrison, and J. Sapirstein, Phys. Rev. A 76, 042508 (2007).

[6] G. S. Adkins and J. Sapirstein, Phys. Rev. A 78, 062503 (2008).

[7] A. O. Barut, J. Math. Phys. 32, 1091 (1991). 
[8] J. W. Darewych, Annales de la Fondation Louis de Broglie (Paris) 23, 15 (1998); Interparticle Interactions and Nonlocality in Quantum Field Theory, J. W. Darewych, in Causality and Locality in Modern Physics and Astronomy, G. Hunter et al. (eds.), Kluwer Academic Publishers, Dordrecht, 1998, pp. 333-344.

[9] J. W. Darewych, Can. J. Phys. 76, 523 (1998).

[10] G. S. Adkins, R. N. Fell, and P. M. Mitrikov, Phys. Rev. Lett. 79, 3383 (1997).

[11] G. S. Adkins, R. N. Fell, and P. M. Mitrikov, Phys. Rev. A 65, 042103 (2002).

[12] G. S. Adkins and J. Sapirstein, Phys. Rev. A 58, 3552 (1998).

[13] G. S. Adkins and J. Sapirstein, Phys. Rev. A 61, 069902(E), (2000).

[14] G. W. F. Drake and M. Grigorescu, J. Phys. B: At. Mol. Opt. Phys., 38, 2005, pp. 3377-3393.

[15] S. Bubin, M. Stanke, D. Kedziera, and L. Adamowicz, Phys. Rev. A 75, 062504 (2007).

[16] D. B. Cassidy and A. P. Mills, Nature (London) 449, 195 (2007).

[17] M. Barham and J. W. Darewych, J. Phys. B 41, 185001 (2008).

[18] J. Mitroy and S. A. Novikov, Phys. Rev. A 70, 032511 (2004).

[19] J. Zs. Mezei, J. Mitroy, R. G. Lovas, and K. Varga, Phys. Rev. A 64, 032501 (2001).

[20] A. G. Terekidi and J. W. Darewych, J. Math. Phys. 46, 032302 (2005).

[21] A. G. Terekidi and J. W. Darewych, J. Math. Phys. 45, 1474 (2004).

[22] A. G. Terekidi, J. W. Darewych and M. Horbatsch, Can. J. Phys. 85, 813 (2007).

[23] E.A.G. Armour, J.-M. Richard, and K. Varga, Physics Reports 413 (2005) 1-90.

[24] E. A. Hylleraas, Z. Phys. 48469 (1928).

[25] E. A. Hylleraas, Z. Phys. 54347 (1929). 
[26] G. W. F. Drake, Phys. Rev. A 18820 (1978).

[27] G. W. F. Drake, High Precision Calculations for the Rydberg States of Helium, in Long-Range Casimir Forces: Theory and Recent Experiments on Atomic Systems, Ed. by F. S. Levin and D. A. Micha. Plenum Press, New York, 1993, p. 107.

[28] G. W. F. Drake, High Precision Calculations for Helium, in Atomic, Molecular and Optical Physics Handbook, Ed. by G. W. F. Drake. AIP Press, Woodbury, New York, 1996, p. 154.

[29] U. D. Jentschura, and G. W. F. Drake, Can. J. Phys. 82 (2004) 103-108.

[30] D. B. Cassidy, V. E. Meligne, and A. P. Mills, Jr., Phys. Rev. Lett. 104, 173401 (2010).

[31] D. B. Cassidy, M.W. J. Bromley, L. C. Cota, T. H. Hisakado, H.W. K. Tom, and A. P. Mills, Jr., Phys. Rev. Lett. 106023401 (2011).

[32] M. Emami-Razavi and J. W. Darewych, J. Phys. G 32, 1171 (2006).

[33] M. Emami-Razavi and M. Kowalski, Phys. Rev. D 76, 045006 (2007).

[34] M. Emami-Razavi, N. Bergeron, J. W. Darewych, and M. Kowalski, Phys. Rev. D 80, 085006 (2009).

[35] M. Emami-Razavi, N. Bergeron, and J. W. Darewych, J. Phys. G: Nucl. Part. Phys. 38, 065004 (2011).

[36] Y. Suzuki and K. Varga, Stochastic Variational Approach to Quantum Mechanical Few-Body Problems (Springer-Verlag, Berlin 1998).

[37] Y. C. Jean, P. E. Mallon, and D. M. Schrader, Principles and Applications of Positron and Positronium Chemistry, (World Scientific, Singapore, 2003), pp. 17-36.

[38] S. J. Brodsky and R. F. Lebed, Phys Rev. Lett. 102, 213401 (2009).

[39] L. Di Leo and J. W. Darewych, Int. J. of Mod. Phys. 16, 2165 (2002). 